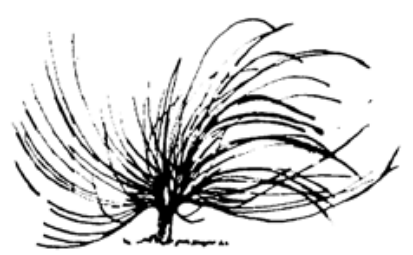

\title{
El giro decolonial en el siglo XXI ${ }^{1}$
}

\author{
Abdiel Rodríguez Reyes ${ }^{2}$ \\ Universidad de Panamá \\ Panamá \\ abdiel.rodriguezreyes@up.ac.pa
}

\section{Resumen}

Este artículo analiza lo que es el giro decolonial en el siglo XXI. La reflexión teórica sobre el giro decolonial es reciente, está vinculada a la población latina en universidades norteamericanas. Aún no hay una definición exacta al respecto ni una comunidad homogénea que se circunscriba estrictamente a ello. Este es un concepto en desarrollo. Es trabajado por un grupo heterogéneo de docentes e intelectuales que conjugan teoría y práctica para darle sentido a una serie de preocupaciones que comparten relacionadas con la crítica de la colonialidad del

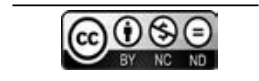

Recibido: 24 de abril de 2016-Aprobado: 22 de setiembre de 2016

1 Este artículo es producto de largas discusiones con el Grupo de Feminismo Decolonial, en el que nos reunimos todos los meses en la librería KaXilda (Gipuzkoa - País Vasco). Agradezco a Nelson Maldonado - Torres por sus comentarios y haberme facilitado parte de la bibliografía.

2 Investigador en la Universidad de Panamá. Estudió Filosofía y Ética en la Universidad de Panamá, Ciencias Sociales en la Facultad Latinoamericana de Ciencias Sociales (FLACSO/ Panamá). Candidato a Doctor en Filosofía por la Universidad del País Vasco. 
poder. Dada la amplitud del tema, me limitaré a mencionar algunos temas, autores y autoras, y bibliografía para tener una idea al respecto.

Palabras clave: Pensamiento, colonialismo, filosofía, cultura dominante, América Latina.

\begin{abstract}
This paper analyzes the Decolonial Turn in the XXI century. The theoretical reflection on the Decolonial Turn is recent and is linked to Latinos at North American universities. There is still no accurate definition for it or a homogeneous community that can be strictly circumscribed to it. This is a developing concept, which is worked out by a heterogeneous group of professors and intellectuals who combine theory and practice to make sense of a series of serious concerns related to the criticism of the coloniality of power. Given the breadth of the subject, I will just mention a few topics, authors, and literature to get an idea about it.
\end{abstract}

Keywords: thinking, Colonialism, Philosophy, dominant cultures, Latin America

\title{
Introducción
}

Vamos a indicar los antecedentes y los tópicos que trata el giro decolonial. Para empezar, podemos decir que un giro significa un cambio de dirección, un golpe de timón; el prefijo (de) significa disociación. A lo largo de la historia hubo diversos giros, como por ejemplo: el copernicano y el pragmático, que supusieron cambios significativos en la forma de comprender el mundo. En tanto, el giro decolonial es un cambio de dirección en la comprensión de la modernidad. Dada la amplitud del tema y la variedad de enfoques con que se ha tratado esta misma cuestión, es imposible no decir que el giro decolonial es una propuesta problemática y divergente con las diversas formas de comprender la modernidad.

El giro decolonial va acompañado de prácticas decoloniales, estas últimas siempre han existido; son un resistirse al orden impuesto 
desde 1492 con la llegada de España al continente de Abya Yala, fecha en que Colón pudo dominar al indígena y hacerse de sus tierras; empezando así una nueva era mundial, la colonialidad. La cual podemos definir como:

Uno de los elementos constitutivos y específicos del patrón mundial de poder capitalista. Se funda en la imposición de una clasificación racial / étnica de la población del mundo como piedra angular de dicho patrón de poder, y opera en cada uno de los planos, ámbitos y dimensiones, materiales y subjetivas, de la existencia cotidiana y a escala social. Se origina y mundializa a partir de América. (Quijano, 2000, p. 342)

Teniendo en cuenta la definición de Quijano (2000), el giro decolonial es una crítica a la colonialidad. Como reflexión teórica es reciente, surge con los pensadores y pensadoras latinas en Estados Unidos (Dussel, et al., 2011), que incorporan nuevos elementos de reflexión transversalmente y se alimenta de otras matrices teóricas y núcleos problemáticos, que veremos más adelante. Es frecuente también utilizar el concepto decolonialidad como sinónimo de giro decolonial, pero este posee más bien connotaciones prácticas de resistencia, en ese sentido tiene al menos quinientos años de existencia, desde 1492 con la implementación de la colonialidad como patrón mundial, ya que desde el encuentro se impuso un sistema de dominación, con dos salidas: asimilación o exterminio. Por ejemplo: los pueblos mexicas vieron como la conquista española destruyó su mundo (León Portilla, 2005).

La crítica no es un reduccionismo de que los grupos indígenas eran buenos y los españoles eran malos, sino, que la organización del Nuevo Mundo para los grupos españoles se hizo en función de las necesidades e intereses colonizadores, no fue una relación recíproca. Tanto así que se modificó la gestión gubernamental de España, ya que se tenía que "obtener una autentica información... sobre la geografía, el clima, la producción y los habitantes de las posiciones españolas en América" (Elliot, 2015, pp. 67-68). Esto demuestra lo importante que era lo que podían extraer. Se tenía que obtener información precisa de los recursos, en particular de los metales preciosos, que escaseaban en Occidente y eran necesarios para pagar las deudas y la acunación de monedas, pieza crucial en lo que sería la configuración del estatus de superioridad 
europea. Así se fueron creando nuevas relaciones de poder, donde las españolas quedaron mejor posicionadas. La nueva relación entre indígena y el sujeto extraño español planteaba la paradoja de dominación - liberación (Dussel, 1974).

La crítica al orden colonial es antiquísima, podría remontarse a Bartolomé de las Casas como el primer crítico de la modernidad (Dussel, 2007). Pero la crítica del giro decolonial no es al colonialismo solamente, en el sentido de que los grupos españoles se establecieron y mantuvieron sus colonias; la crítica es a la paradoja dominación - liberación que nos plantea Dussel (1974) y a la colonialidad del poder que nos plantea Quijano (2000), esto último como un nuevo patrón de poder mundial con base en la explotación/dominación/conflicto que se mundializa a partir de 1492 y se mantiene hasta la actualidad con considerables variaciones, pero con el mismo telón de fondo.

\section{Antecedentes}

Hacer un recuento total de los antecedentes del giro decolonial resulta imposible para un artículo. Sin embargo, hay algunas cuestiones que no se pueden pasar por alto. Pasaremos revisión a los antecedentes claves que son el sustento teórico del giro decolonial; podemos decir que es una empresa interdisciplinaria, por lo que no se puede limitar a un solo saber, toma aspectos de varias disciplinas y diversos enfoques. Por lo amplio del tema, solo consideraremos algunos puntos claves que se pueden tomar como antecedentes, donde tema tras tema se va entroncando con otro. El giro decolonial irá tomando aspectos de cada uno de estos antecedentes.

\section{La filosofía de la liberación}

La filosofía de la liberación surge a finales de la década del sesenta. No es un movimiento filosófico unitario. En 1976 Dussel (2011) escribió un libro de ideas sentenciosas cuyo título fue Filosofia de la liberación, donde expresó pioneramente la necesidad de una filosofía posmoderna, no en el sentido de Lyotard, de solo debilitar la razón moderna, sino como un intento de superación de la filosofía moderna; en esta ocasión no me referiré a esta problemática. Hay otros libros anteriores de Enrique Dussel que fueron marcando los núcleos problemáticos 
que queremos resaltar aquí como antecedentes, por ejemplo: Historia de la iglesia en América Latina. Coloniaje y liberación 1492 / 1973 (Dussel, 1974). Es un libro poco citado, pero sumamente interesante. En el texto podemos enumerar varios aspectos, parte de la historización de la primera institución en el Nuevo Mundo, lo que brinda información del hecho histórico, en particular de la contraposición dominación - liberación que surge a partir de este, en tres niveles: geo-político, erótico y pedagógico. A nivel geo-político, igual que las teorías latinoamericanas de la dependencia, concibe la organización del mundo en centro y periferia, que consiste en exportar metales preciosos para Occidente, lo que permitió la acuñación de monedas que escaseaban, esto significó un trampolín para el desarrollo de Europa. Con el "descubrimiento" del Mar del Sur, se completará el mercado mundial, lo que fue importante para el desarrollo del capitalismo, en donde tener el control de la ruta de tránsito entre el Pacífico y el Atlántico traía beneficios que no eran precisamente para los pueblos indígenas. A nivel erótico, analiza la nueva realidad interpersonal a partir del encuentro, se impone una sociedad patriarcal con doble moral; la libido impulsa al varón español a tener sexo con la indígena (por su desnudez) obligándola (violación) y nada se lo impide; pero a su esposa le pide castidad, que esté en la casa; esta es la dominación a nivel erótico (sexual) de la mujer. A nivel pedagógico, España colonizó al indígena, lo dominó con las armas y le impuso su cultura, la forma de pensar española occidental se interiorizó en el yo personal y social indígena. Un último punto interesante que está a lo interno es el «yo conquisto» que se puede interpretar como el "yo pienso" del sujeto conquistador español ante el conquistado que no piensa porque es un bárbaro.

\section{Análisis de sistemas - mundo}

El fundador de esta perspectiva o movimiento intelectual es Immanuel Wallerstein, y el epicentro de difusión fue el Fernand Braudel Center de la Universidad Estatal de Nueva York. Esta perspectiva parte también como las teorías de la dependencia (Dos Santos, 2002), de la existencia del centro y la periferia, incluyendo, además, la semiperiferia; así se configuró el moderno sistema mundial con este esquema. El texto central es el tomo I del Moderno sistema mundial de Wallerstein (1974), que parte del siglo XVI cuando se formó la economía mundial 
con el "descubrimiento" de Abya Yala y, consecutivamente, la constitución del capitalismo como sistema global.

Otra cuestión que integra es la geocultura como marco cultural del moderno sistema mundial que consiste en que la ciencia y el conocimiento sui géneris son productos europeos y norteamericanos. Desde esta perspectiva se rescata también el estudio de los fenómenos a nivel civilizatorio en clave braudeliana de larga duración, lo que brinda una visión y crítica histórica de los cambios y las crisis civilizatorias. Esto da, a la misma vez, una visión amplia y abstracta, pero se concreta en los estudios de las periferias y semiperiferias y las regiones en particular (estudios de caso aplicando esta perspectiva), midiendo, entre otras cosas, el uso de las materias primas y el trabajo de los países dependientes, y las relaciones asimétricas entre estados. A nivel social se constituye también el racismo, la etnicidad y el sexismo; lo que trae como consecuencia la discriminación. Ante las diversas manifestaciones en contra del sistema, la perspectiva de análisis de sistemas-mundo acuña el concepto de "movimientos antisistémicos", para dar cuenta de la serie de movimientos y movilizaciones que no solo buscan llegar al poder etc....si no, propuestas que están en contra del sistema (como el EZLN), lo que acuerpa aquel lema del Foro Social Mundial: "otro mundo es posible".

\section{Estudios chicanos}

El movimiento de la población chicana emerge a partir de la segunda mitad del XX. Pero no nos referiremos a todo lo relativo a este movimiento. Sino, más bien, a sus puntos claves que nos ayuden a saber cómo se va formando lo heterodoxia en las practicas intelectuales latinas en Estados Unidos, que muy bien retoman el giro decolonial. Un elemento cohesionador de los diversos movimientos (negros, latinos, hippies y otros más), fue la guerra contra Vietnam, esto nos da una pista de que diversos movimientos pueden converger por una causa, en el caso citado, contra la guerra. En cuanto al movimiento chicano propiamente, varios elementos confluyen para que se active un movimiento de esta naturaleza, tanto en el plano político (activismo y militancia) como en el intelectual; podríamos decir que la discriminación racial, las enormes adversidades que tienen que enfrentar, y la creciente tendencia demográfica de los grupos mexicanos en Estados Unidos despertó en 
la sociedad conservadora norteamericana un sentimiento de xenofobia; donde Donald Trump es la expresión más clara de ello. Un resistirse a eso, es el movimiento chicano. Y, a esto que se resiste no solo afecta a su población, sino a la población migrante sin recursos (latinoamericana, india, africana, oriental) que tiene como destino a Estados Unidos en busca de un mejor futuro (american dream). Los grupos chicanos pelearon por sus ideas, entre las cuales caben destacar que estaban en contra del asimilacionismo, es decir, que su cultura sea subsumida por la cultura hegemónica norteamericana. Esto llevó al movimiento a optar por su propia dinámica educativa, a rescatar su historia, a que en los programas educativos se reflejara su experiencia, sus conocimiento y su vivencia; todo ello en el marco de una sociedad tan contradictoria como la norteamericana. Peticionar que se abrieran espacios para los estudios chicanos propiamente es uno de los pilares centrales del movimiento (Salas y Valle, 2002). Estas reivindicaciones simultáneamente darían paso a la necesidad, en términos macros, de abrir espacios más amplios, los cuales se lograron a partir de las insurgencias estudiantiles; de allí nacen los estudios étnicos (Grosfoguel, 2007). Esto quiere decir que no son ningún privilegio, estos departamentos nacen al calor del tropel de estudiantes que buscan espacios académicos que respondan a sus propios núcleos problemáticos. No es coincidencia que quienes inician el giro decolonial sean profesores y profesores de departamentos precisamente de estudios étnicos (de las universidades norteamericanas), como Ramón Grosfoguel y Nelson Maldonado-Torres, entre otros.

\section{Estudios poscoloniales}

Antes de analizar las tesis de los estudios poscoloniales, es necesario mencionar que los estudios subalternos (Guha y Spivak, 1988) y los estudios culturales son el motor de arranque de los estudios poscoloniales. La razón es muy sencilla; son los grupos pensadores indios en universidades angloparlantes que se identificaron en un primer momento con los estudios subalternos, los que posteriormente integrarían lo que se conoce como estudios poscoloniales. Partiendo del sujeto subalterno, el que es como un ser humano de menor rango, así como en la jerarquía militar; evidencia el carácter político de los estudios subalternos (Chakrabarty, 2008), donde el sujeto subalterno siempre está en tensión. Sin embargo, este carácter político - según los críticos como Ella 
Shohat por ejemplo - se desdibuja en los poscoloniales, porque no está la tensión que estaba muy clara en los subalternos. Como hemos señalado, tuvo su acogida principalmente en universidades angloparlantes, en los departamentos de Estudios Culturales y Literatura Comparada. En estos estudios se puede constatar la enorme influencia de fuentes filosóficas francesas. Este es uno de los aspectos a contrastar con el giro decolonial, ya que, siguiendo a Mignolo:

El pensamiento decolonial se diferencia de la teoría poscolonial o de los estudios poscoloniales en que la genealogía de estos se localiza en el posestructuralismo francés más que en la densa historia del pensamiento planetario decolonial. (Mignolo, 2007, p. 661)

También hay que tomar en cuenta la heterodoxia de los estudios poscoloniales que desentrañan en la literatura "universal" los ribetes de colonialismo. Como, por ejemplo, las clases en Harvard de H. Bhabha van en esa dirección, en donde la literatura juega un papel importante en el imaginario de integrar, en una red global, las diversas culturas, en el contexto del imperialismo. Un conocimiento de esta naturaleza tiene un efecto crítico para una comprensión de la modernidad diferente, en donde el colonialismo es la mano derecha del imperialismo y, la literatura se instrumentaliza como herramienta de difusión y legitimadora de este mismo. Cabe resaltar, aquí, algunas diferencias y similitudes con el giro decolonial. Los estudios poscoloniales surgen de otro contexto, aunque con el mismo telón de fondo. Parten, en un primer momento, de la experiencia india. Pero ya va más allá la crítica, ya no solo está en el contexto de la India, ni solo de la diáspora hacia universidades norteamericanas e inglesas (también en Australia como el caso de Dipesh Chakrabarty y Ranajit Guha que tenían estancias en universidades australianas). Lo neurálgico hoy de estos estudios es que centran la atención en el anverso y reverso del sistema colonial, como sus consecuencias. Como diría Stuart Hall:

[Los estudios poscoloniales] nos está[n] obligando a reinterpretar la propia forma binaria en la que se ha representado durante tanto tiempo el encuentro colonial. Nos obliga[n] a reinterpretar las divisiones binarias como formas de transculturación, de traducción 
cultural, abocadas a perturbar para siempre las divisiones binarias culturales del aquí y el allí. (Hall, 2008, p. 128)

Es decir, que no es un problema local, sino global, y que los análisis del entronque cultural simplistas binarios se caen sobre su propio peso. De lo que se trata, y eso es uno de los meritos de los estudios poscoloniales, es de ver cuestiones que antes no se veían o no se tomaban en cuenta (más adelante resaltaremos algunos ejemplos) -Charles Taylor diría sobre un libro de Chakrabarty (2008) que su merito es sacar a la palestra todo aquello que normalmente se pasaba por alto-, agregaría que sacar a la palestra lo que no forma parte de los núcleos problemáticos del pensamiento canónico. La crítica no es estar en pro o contra del colonialismo o colonialidad, sino la interacción de las culturas en el contexto de la expansión colonial de los imperios (en el transcurso de la historia) y las consecuencias de esto. En cuando a las similitudes, tanto en lo decolonial como en lo poscolonial confluye la crítica del dominio y el discurso colonial establecido por los imperios. Con la diferencia de que parten de experiencias concretas distintas.

\section{Marxismo y feminismos negros}

Es muy importante que los propios sujetos afectados tomen la palabra y enuncien su propia crítica. Los movimientos sociales despiertan movimientos intelectuales; ambas cuestiones van de la mano, son interdependientes. Esto es una realidad poco objetable, en la que quien sufre alguna afección tiene derecho a enunciar una crítica desde su particular situación, así las críticas situadas se deben integrar para dar forma al discurso crítico, ya con la diversidad de críticas integradas, pero sin perder su especificidad en el campo material de la vida social y política. Como hemos visto con otros ejemplos, Estados Unidos es el país de las contradicciones. Es donde habita el Imperio, es donde encontramos riqueza y pobreza al extremo. En cuando al surgimiento de diversos pensamientos negros, no es la excepción. Solo hay que recordar los trabajos pioneros y la praxis política de Angela Davis, que tienen una fuerte carga de filosofía (estudio con Marcuse y Adorno), entrecruza la raza, la clase y el género (Davis, 2005); y cómo el complejo carcelario industrial (Davis, 2016) se convierte en un dispositivo de castigo racial entre otras cosas. 
Pero particularmente queremos resaltar primero el trabajo de Robinson (2000) de marxismo negro y segundo el trabajo de Hill (2000) de feminismos negros. En cuanto la raza, se convirtió en un elemento de segregación racial. Robinson, que escribió Black Marxism en 1983, señaló que el capitalismo es racial, es decir, que la segregación racial es constitutiva del capitalismo como forma de organización de la sociedad. Lo que representó la explotación (esclavitud) de la población negra, la segregación (apartheid) y la marginalización a determinadas zonas, en gran parte sin seguridad social garantizada. En otros términos, la raza determinó la clase social de esta parte importante de la población humana.

En cuanto a los feminismos negros, habría que decir que aportan miradas de las propias afectadas por su condición de raza y género. Esto tiene que ver como muchos de los temas tratados aquí, con una insurgencia epistémica, un estar en contra de los saberes hegemónicos; en este sentido, las feministas negras toman distancia del feminismo producido en la academia (feminismo hegemónico) que no puede encarar o no comprende los problemas particulares de raza y clase, sino que solo se centra en la cuestión de género. Este es un proceso que se va afianzando, y como bien señala Hill (2000) que en momentos parecerá difuso, pero con el andar y que es un feminismo que responde a un problema en concreto, tendrá sentido, además de enunciar su crítica de lo que viven, articulándose con otros sectores o movimientos sociales contra-hegemónicos.

Los feminismos negros (Jabardo, 2012) plantean el tema de que no solo hay un feminismo sino varios; esto también lo veremos en las Epistemologías (De Sousa Santos y Meneses, 2014) y Filosofias del sur (Dussel, 2015); la constante es apelar a la diversidad, antes que a la unidad abstracta universal de un (único) discurso. Una de las cuestiones de mayor peso de esto que hemos visto es la posibilidad de diversidades de discursos críticos que se articulan analógicamente entre sí.

\section{Epistemologías del sur}

El principal teórico de la Epistemología del sur es Boaventura de Sousa Santos. En la actualidad, el proyecto Alice materializa esta epistemología, con escuelas de verano, conferencias, coloquios, conversaciones alrededor del mundo con intelectuales y activistas que dan cuenta de diferentes perspectivas. Se publicó un libro con el título de 
este epígrafe hasta el 2009, pero ya con anterioridad Boaventura venía trabajando los temas que componen lo que es Las epistemologías del sur. De forma innovadora Boaventura articula ideas en un primer texto: Una epistemología del sur, pero brevemente en un compendio lo hace en plural: Epistemologías del sur (Santos y Meneses, 2014), dando cuenta de que no solo existe una epistemología del sur, sino varias. Este es un punto muy importante; recientemente Dussel (2015) publicó en la misma línea: Filosofías del sur. Otra idea interesante que desarrolla Boaventura, es la de sur global, que son todas las periferias de las antiguas colonias, e incluso la ida de sur llanamente denota menor desarrollo que el norte (por ejemplo, el norte de América está más desarrollado que el sur, como también en Europa pasa lo mismo, los países del Norte gozan de mayor bienestar). A partir de estas tensiones, se articulan preocupaciones epistemológicas y sociales que son fuentes de conocimiento no siempre tomadas en consideración; lo que nos lleva a plantearnos que no se puede hablar de justicia social sin la epistémica, esto último es uno de los aspectos más brillantes en Boaventura y las epistemologías del sur.

En esta misma línea, la ecología de los saberes problematiza la idea de un saber universal supra. Parte más bien de la existencia de otros saberes que son invisibilizados, esto es una crítica a lo llamado la monocultura y el rigor científico, en donde estos otros saberes quedan al margen del conocimiento universal supra; pero, en la ecología de los saberes, son validos igual que los occidentales; el problema consiste en que como son ignorados, da la sensación de no tener validez, como sí la tiene el conocimiento científico occidental, que es difundido por las universidades canónicas. La ecología de los saberes cuestiona la preeminencia de un solo saber ante la multiplicidad de saberes, por eso opta por el pluralismo epistemológico. Hay que tomar en consideración que las epistemologías del sur también es un proyecto político emancipador que busca reinventarse el poder. Por eso el acompañamiento de Boaventura a los movimiento sociales anti-globalización como un intelectual de retaguardia.

\section{Sobre el giro decolonial}

Con este breve recuento de los antecedentes del giro decolonial, hemos visto cómo todas estas teorías y perspectivas le confieren 
preponderancia a la diversidad, a la heterogeneidad y a que los campos de estudio tengan un impacto en lo social y político. Este es un punto fundamental del cual no se tiene que prescindir. La crítica no solo es teórica, sino también contempla un cambio social y político. El giro decolonial está muy vinculado a la población latinoamericana en Estados Unidos. Como concepto propiamente, es introducido y desarrollado por Maldonado-Torres en el artículo titulado "Césaire's Gift and the Decolonial Turn" que apareció en el 2006 en la Radical Philosophy Review. Esto no se puede analizar desasociado de la gran ascendencia del movimiento latino en la agenda política estadounidense; son más de 50 millones de personas latinas en Estados Unidos y aproximadamente el $50 \%$ está registrada para votar en las elecciones.

$\mathrm{Al}$ igual que los grupos pensadores indios, los latinos tienen presencia en las universidades norteamericanas de mayor prestigio. Hay que ver la cartografía de la diáspora (Brah, 2011) donde se verá mucho el movimiento de pensadoras y pensadores de India y Latinomérica en universidades de Estados Unidos, no exclusiva, pero sí significativamente. El giro decolonial sui generis está relacionado con una red heterogénea de pensadoras y pensadores que se está ampliando cada vez más, fundamentalmente en universidades y escenarios angloparlantes; así, Estados Unidos e Inglaterra llevan un paso adelante en este campo de estudio con respecto al resto de las universidades. No es casualidad - según el Ranking Web de Universidades - que las "mejores" universidades del mundo se encuentran en Estados Unidos e Inglaterra. Es desde estas grandes universidades que los grupos pensadores latinoamericanos lanzan lo que se conoce como el giro decolonial, cuestión que ya no solo se limita al ámbito institucional universitario, también tiene un impacto en el activismo político: Lo que podríamos llamar las prácticas políticas decoloniales.

Quiero llamar la atención sobre dos textos (compilaciones) centrales para comprender lo que es el giro decolonial. Son el resultado de encuentro en el norte de América (Canadá y Estados Unidos), ambos textos articulan las preocupaciones que van a ser constantes en este giro. Si bien no se agotan aquí, es necesario resaltar que son de los textos que explícitamente responden, articulan y desarrollan la problemática de la "dominación - liberación" planteada por Dussel (1974) y la colonialidad como uno "de los elementos constitutivos y específicos del 
patrón mundial de poder" de Quijano (2000, p. 342); además de estar en diálogo con los antecedentes que ya vimos en epígrafes anteriores.

El primer texto es La colonialidad del saber: Eurocentrismo y ciencias sociales (Lander, 2000). Esta compilación tenía como núcleo problemático medir el impacto del eurocentrismo en las ciencias sociales, para así analizar la relación con el colonialismo. Fue el resultado de un simposio titulado: Alternativas al eurocentrismo y colonialismo en el pensamiento social latinoamericano contemporáneo en el marco del Congreso Mundial de Sociología en Montreal en 1998. El texto analiza el desarrollo de la colonialidad y el papel que está jugando en la actualidad el neoliberalismo; teniendo en cuenta el presente estado de cosas que oculta la colonialidad del poder:

Esta fuerza hegemónica del pensamiento neoliberal, su capacidad de presentar su propia narrativa histórica como el conocimiento objetivo, científico y universal y a su visión de la sociedad moderna como la forma más avanzada -pero igualmente normal- de la experiencia humana, está sustentada en condiciones histórico culturales específicas. El neoliberalismo es un excepcional extracto, purificado y por ello despojado de tensiones y contradicciones, de tendencias y opciones civilizatorias que tienen una larga historia en la sociedad occidental. Esto le da la capacidad de constituirse en el sentido común de la sociedad moderna. (Lander, 2000, p. 12)

Al constituirse de esta manera la sociedad moderna en la que vivimos, es muy difícil cuestionarla, ya que esta en nuestra psique la idea que este es el único mundo posible y que transformarlo es una chapucería. El pensamiento neoliberal garantiza este estado de cosas autenticando los saberes coloniales que son funcionales al capitalismo. Los textos que integran esta compilación son una reflexión histórica que responde prospectivamente. Nuestra forma de comprender y actuar hoy es el resultado de la colonialidad del poder, el saber, la vida, incluso hasta lo espiritual se configuró en torno a ello.

El segundo texto Giro decolonial (Castro-Gómez y Grosfoguel, 2007) fue el resultado de otro encuentro, también en Estados Unidos. No es casualidad que la mayoría de los encuentros se desarrollen en Estados Unidos (Duke, Binghamton, Berkeley), sin duda, es el país de 
las contradicciones desde donde se puede desarrollar un pensamiento de esta naturaleza. La competitividad de estas universidades y el impacto que tienen a nivel global ayudaron a dar a conocer las preocupaciones del giro decolonial. Las universidades que no se ocupen de esta temática quedarán fuera del debate del pensamiento crítico global. En la actualidad las inmigraciones masivas, el racismo y la desigualdad siguen aumentando indiscriminadamente; el giro decolonial estudia estos fenómenos que nos carcomen como consecuencia de la colonialidad del poder.

Se da por sentado que con el fin del colonialismo estándar se acabarían los problemas. Lo cierto es que ocurre todo lo contrario, en esta nueva etapa la que el colonialismo se quedó sin legitimidad (aunque aún persiste concretamente), se articulan nuevas formas de dominación extra-territorial. Y, ser dependiente del Imperio, que es el que puede colonizar, no necesariamente trae beneficios, por ejemplo: El Estado Libre Asociado de Puerto Rico vive una profunda crisis económica que además intenta arrodillar las intensiones soberanistas. Otro ejemplo es Panamá que puso fin al enclave colonial con el Tratado Torrijos-Carter, pero aún tiene otro, el Tratado Concerniente a la Neutralidad Permanente y Funcionamiento del Canal de Panamá a perpetuidad, en el que Estados Unidos puede intervenir militarmente cuando la neutralidad del Canal se vea expuesta, es decir, persisten las formas de mantener el control en los antiguos espacios coloniales. Así, consecutivamente, se puede ver que la colonialidad del poder no baja la guardia, más bien afina sus dispositivos para mantener el control.

En la actualidad, en nombre del progreso y los discursos oficiales se dan muchas cosas por solucionadas o aisladas, pero no es así:

La división internacional del trabajo entre centros y periferias, así como la jerarquización étnico-racial de las poblaciones, formada durante varios siglos de expansión colonial europea, no se transformó significativamente con el fin del colonialismo y la formación de los Estados-nación en la periferia. Asistimos, más bien, a una transición del colonialismo moderno a la colonialidad global, proceso que ciertamente ha transformado las formas de dominación desplegadas por la modernidad, pero no la estructura de las relaciones centro-periferia a escala mundial. (Castro-Gómez y Grosfoguel, 2007, p. 13) 
La colonialidad global es cuando en todas partes del mundo se viven injusticias, por ejemplo: la indigencia y la pobreza aumentan, mientras que la riqueza se concentra en el 1\% de la humanidad. Aún la periferia está subordinada social, cultural y epistémicamente a los países del centro industrializados. Dada la complejidad de la afectación de la colonialidad del poder, las nomenclaturas utilizadas cada vez son más extensas para dar cuenta de todas las atrocidades cometidas. La barbarie del siglo pasado re-aparece con una nueva careta. Hay poca diferencia entre un judío en un campo de concentración nazi y un inmigrante en un campo de refugiados en Turquía. Los derechos humanos se desvanecen en el aire.

El concepto (en construcción) propiamente de giro decolonial es desarrollado, como hemos dicho, por grupos latinoamericanos, no exclusivamente, en parte por vivir la experiencia material por varios siglos de estar bajo un sistema colonial, igual que gran parte de Asía, Oriente y África, lo que permite tener una visión concreta de la cuestión. Así como hay intentos de liberación, es necesario tener en cuenta que la colonialidad tendrá su contraofensiva en todas las dimensiones de dominación; que tratará de re-acomodarse en el siglo XXI; por lo que las respuestas también deben ir en la dirección de poder contrarrestarla.

En el texto Giro decolonial está concentrada gran parte de las tesis de este enfoque interdisciplinario. A partir de este texto ya se menciona al pie de la letra este concepto (en construcción). Mignolo (2007), en una pequeña historia de El pensamiento decolonial hace referencia a la relación modernidad/colonialidad como elementos insoslayables; además añade elementos de la instrumentalización (teoría crítica). Aquí habría que añadir que el giro decolonial no tiene que renunciar a un diálogo con la teoría crítica (Insausti, 2015), lo que habría que preguntarse es hasta qué punto estarán abiertos al diálogo. Siguiendo con Mignolo, explica la cuestión del giro como cambio de dirección para comprender e interpretar el mundo en diferentes momentos. En este sentido, la idea de colonialidad del poder expuesta por Quijano (2000) abrió paso a ese giro. La idea de desprenderse de la episteme que surgió con el proceso colonial hacia una apertura, a una verdadera intercomunicación entre culturas, es el proceso que "está en marcha" (Mignolo, 2007, p. 45).

En esta misma línea de ideas, Maldonado-Torres (2011) señala:

Utilizaremos los conceptos de giro descolonial y de giros descoloniales para dar cuenta del carácter internacional y del significado 
de formas de pensamiento que se inspiran simultáneamente en la crisis del pensamiento y del proyecto de civilización europeo por un lado, y en la afirmación de posibilidades de ser, de poder, y de conocer que superan los límites constitutivos de la modernidad eurocéntrica, por otro. Así como ha habido giros copernicanos, pragmáticos, lingüísticos, y posmodernos, de forma análoga se puede identificar un giro descolonial o giros descoloniales, conceptos que se refieren a cambios fundamentales en las coordenadas básicas del pensamiento. (Maldonado-Torres, 2011 p. 683)

Nótese que también se plantea la cuestión en plural, por el pluriverso de giros que se pueden ir dando en función del rechazo de la razón colonial unívoca. A la par de todos estos elementos teóricos, están las prácticas decoloniales, que rompen con el status quo, por ejemplo: las poblaciones inmigrantes piden respeto, las mujeres piden semejanza, las parejas del mismo sexo piden el reconocimiento civil de su unión. Se hacen diversas performance muy creativas que expresan de forma innovadora las afecciones de estos movimientos. El giro decolonial articula teóricamente lo que en la práctica luchan miles de personas, no de la misma forma, tampoco con la misma intensidad, pero sí compartiendo analógicamente el hecho material de ser víctima de un sistema perverso.

\section{Crítica a la colonialidad del poder}

Como hemos señalado, el giro decolonial es un fenómeno relativamente reciente que se podría interpretar, como hemos visto, como una crítica a lo que Quijano (2000) llamó la colonialidad del poder; planteamiento que cuestiona las relaciones de poder y la clasificación social; centrando la atención en la explotación/dominación/conflicto principalmente en la "producción social del género" y "las diferencias fenotípicas de la categoría de raza" (Quijano, 2000, p. 373), entre otras cuestiones. Esto fue planteado a finales del siglo pasado y en el siglo XXI necesita ser re-planteado; empero, sin olvidar su matriz histórica. El propio Quijano sigue haciendo esfuerzos significativos para explorar las nuevas formas de colonialidad del poder. Pero para los fines de este artículo, resaltaré tres críticas ([a la colonialidad del] saber, género y ser). Aquí crítica en su sentido estricto, de estudio de la cuestión. 


\section{La colonialidad del saber}

La crítica a la colonialidad del saber de Lander (2000), que compiló un texto muy sugerente al respecto que podríamos considerar como uno de los primeros esfuerzos colectivos que se ocuparon de la cuestión propiamente. Centra la atención en que los saberes modernos se articularon para legitimar la organización y las relaciones de poder, en la actualidad con el neoliberalismo como su forma coyuntural de organización societal; este se presenta como un elemento omnipresente, natural e irrefutable. Lander (2000) considera que también es necesario cuestionar la serie de conocimientos que conforman las ciencias sociales que son instrumentalizados a los intereses económicos del orden vigente.

La crítica a la colonialidad del saber o saberes coloniales consiste en evidenciar que estos saberes se articulan con la organización del poder para legitimar cualquier forma de dominación y oprimir cualquier intento de liberación. Como gran parte de las cuestiones tratadas en el Giro decolonial parte del encuentro con España, acontecimiento histórico que fundó por un lado la organización del mundo, pero también simultáneamente "la constitución colonial de los saberes" (Lander, 2000, p. 16). El mundo colonial se industrializó en el siglo XVIII y la ciencia en general y las sociales en particular se constituyeron a partir de esta realidad, fue entonces desde los países liberales europeos desde donde emergieron las ciencias sociales; lo que conocemos como el conocimiento científico con validez universal, a pesar de que respondiera al naciente proceso de industrialización de la Europa central y no necesariamente del mundo en general.

\section{La colonialidad del género}

En cuanto a las cuestiones de género, la crítica a la colonialidad del género de Lugones (2008) concentra la atención sobre la violencia de género en la colonialidad del poder. Propone cuestionar desde la misma constitución del sistema colonial, "la reducción del género a lo privado, al control sobre el sexo y sus recursos y productos" (Lugones, 2008, p. 93). El proyecto civilizatorio de la conquista también representó una transformación del modo de vida de la indígena. La nueva organización social del mundo colonial como proceso de racionalización y sexualización también incluía a la indígena como objeto de dominación 
en cuanto a su raza y su sexo. Metodológicamente, es necesario integrar la otredad y la diferenciación colonial para la investigación en feminismo decolonial (Curiel, 2015). La colonialidad del género, al estudiar la transversalidad de la raza, clase y género, focaliza la triple dimensión que la indígena en cuanto a su raza es invisibilizada, explotada en cuanto a su clase, y como mujer era inferior, según el estereotipo del mundo moderno occidental. Deconstruir esto no es aislado de la misma deconstrucción de la colonialidad del poder. La crítica a la colonialidad del género tiene intrínseco un componente práctico que debe conducir integralmente a la liberación de la mujer.

\section{La colonialidad del ser}

Otra propuesta interesante es la crítica a la colonialidad del ser de Maldonado-Torres (2007). Esta es la crítica ontológica. Contrapone la ontología de Levinas con la de Heidegger. La ontología y el proyecto heideggeriano se comprometió aceptando el rectorado en la Universidad de Friburgo (Heidegger, 1989) con el líder - Hitler - en la conducción de un pueblo que sería el auténtico ante el resto que no lo era; para esto, que ya tiene su antecedente en la colonización de América, en cuanto la vida del otro no tiene valor alguno, era imperante una ontología que saliera de este esquema (instrumental) y tomara en cuenta la cosmología de los pueblos colonizados.

Si la ontología de Heidegger se vinculó al poder, entonces habría que recurrir a una ontología de la víctima del poder, en el humanismo del otrou otra (Levinas, 2005), por ejemplo. Para Maldonado- Torres la clave para entender la crítica decolonial o a la colonialidad del ser es el Cogito ergo sum de Descartes y la autenticidad de Heidegger:

Tanto en la filosofía de Descartes como en la de Heidegger. Si el ego cogito fue formulado y adquirió relevancia práctica sobre las bases del ego conquiro, esto quiere decir que "pienso, luego soy" tiene al menos dos dimensiones insospechadas. Debajo del "yo pienso" podríamos leer "otros no piensan", y en el interior de "soy" podemos ubicar la justificación filosófica para la idea de que "otros no son" o están desprovistos de ser. (Maldonado-Torres, 2007, p. 144) 
La crítica ontológica es la más difícil por los niveles de abstracción; una lectura invertida de la ontología del canon occidental sería develar su lado oscuro, por ejemplo, la lectura de Maldonado- Torres sobre la máxima cartesiana traería a colación una negación del ser indígena, eso es "la ausencia de "ser" en sujetos racializados" (Maldonado-Torres, 2007, p. 145). Como el negro esclavo, como el indígena ante el colonizador, como el judío en un campo de concentración. El pensamiento moderno occidental no críticó al colonialismo sino que lo vio como adherente a su proyecto de emancipación, sin medir el coste humano, donde la discriminación ontológica es la más profunda. Como el propio Maldonado- Torres señala, esta crítica esta en desarrollo, por lo que requiere una lectura de la tradición filosófica occidental en clave decolonial para develar su lado oscuro ontológico. No en el sentido de que quienes se dedican a la filosofía son una especie de Darth Vader, sino que muchas veces sus formas de pensar son develadoras por un lado; pero por el otro, ocultan superioridad y dominación.

\section{Pedagogía decolonial}

Después de ver los antecedentes, los rasgos propios del concepto giro decolonial y la crítica a la colonialidad del poder; ahora nos podemos pasar al tema de cómo seguir aprendiendo y como compartir. Este epígrafe bien podría llamarse metodología y pedagogía decolonial, pero ambas cuestiones, si bien son relacionales, tienen su propia dinámica; en cuanto, a lo metodológico podríamos cuestionar el cómo investigamos, para lo que es clave, y en eso concuerda Catherine Walsh y Nelson Maldonado-Torres, el cambio de actitud. Creo que eso es fundamental, si todavía tenemos la actitud arrogante de que el conocimiento que surge del giro decolonial es inferior o trillado con respecto al conocimiento moderno "universal", poco se avanzará y se aprenderá de este, porque la actitud determina nuestro accionar; en el caso que sea negativa, entonces, se manifiesta como un rechazo al giro decolonial. La cuestión metodológica es importante, y ya hace algunos años se viene trabajando en esta dirección, como la metodología de los grupos oprimidos (Sandoval, 2000), o también, en esta dirección, una descolonización de las ciencias sociales (Bautista, 2012), y una descolonización de la metodología (Tuhiwai, 1999). 
Por el momento nos queremos concentrar en el cambio de actitud pedagógica. Después de pasar una revisión mínima a lo que es giro decolonial, es necesario plantearse la cuestión a nivel pedagógico, así como la Pedagogía del oprimido (Freire, 1992). Ahora bajo las nuevas y sofisticadas formas de dominación en el siglo XXI; el Big Brother de Orwell se hizo realidad y los mecanismos psico de dominación son cada vez más eficientes (Han, 2014). Nos controlan desde una compleja red de información que exterminó la privacidad y nos desnudó ante el mercado. Ante esa realidad inconmensurable de deshumanización, es necesario pensar nuevamente a nivel pedagógico. En su momento, Freire lo hizo en contraposición a la concepción bancaria de la educación, donde se planteaba que hay que trabajar:

En tanto hombres o pueblos en la lucha permanente de recuperación de su humanidad. Pedagogía que haga de la opresión y sus causas el objeto de reflexión de los oprimidos, de lo que resultará el compromiso necesario para su lucha por la liberación, en la cual esta pedagogía se hará y rehará. (Freire, 1992, p. 41)

En el giro decolonial, la pedagogía parte del sujeto oprimido como en Freire, pero con la particularidad de estar en contra de la concepción colonial de la educación. Muy bien anotó Freire que se necesita una pedagogía del sujeto oprimido en un sistema capitalista; pero la crítica es más amplia, sí la hacemos en el marco de la colonialidad; a esto apunta la pedagogía decolonial:

La pedagogía y lo pedagógico aquí no están pensados en el sentido instrumentalista de la enseñanza y transmisión del saber, tampoco están limitadas al campo de la educación o los espacios escolarizados. Más bien, y como dijo una vez Paulo Freire, la pedagogía se entiende como metodología imprescindible dentro de y para las luchas sociales, políticas, ontológicas y epistémicas de liberación. (Walsh, 2014, p. 13)

El significado estándar de que la pedagogía se ocupa de la enseñanza, educación y la trasmisión de conocimiento no funciona para el giro decolonial. Más bien hay que reinventarse una nueva pedagogía para nuevos tiempos. Por eso hay que partir del sujeto oprimido (Freire, 1992) 
que busca la liberación (Dussel, 1980). Con este horizonte, entonces, entretejer alternativas decoloniales; una pedagogía o pedagógica latinoamericana como diría Dussel (1980) con el propio actor, el sujeto, el ser humano, con las colectividades en su accionar liberador. No solo basta trasmitir conocimiento, sino cuestionarlo desde su propia génesis.

Esta muy bien la crítica del giro decolonial y la producción del conocimiento en esa dirección. Pero también es necesario compartir ese conocimiento y seguir aprendiendo de la praxis de la gente, de las colectividades, para eso es lo pedagógico, no puede haber un divorcio entre ambas cosas. La pedagogía decolonial trata de "subvertir la colonialidad" (Walsh, 2014, p. 18). La univocidad del pensamiento moderno y la colonialidad del poder matan la pluriversidad epistémica y anulan el conocimiento que surge de las praxis. Esto no lo excluye ni anula el sector político "profesional" conservador del status quo, el cual se encarga de recortar y no apoyar esto, que es lo que generalmente pasa. El propio conocimiento moderno produce, en su interior, un dispositivo que mantiene al margen lo que atente contra su superioridad, para eso Bautista (2012) señala que hay que emprender un largo viaje de descolonización de las ciencias sociales y también de la filosofía. La alternativa tiene que subvertir esta condición con un cambio de dirección pedagógica hacia una relación más activa, comprometida y de reciprocidad entre la reflexión teórica con la praxis.

\section{A modo de conclusión: Viejos problemas que persisten}

Como esto es una discusión reciente, aún no hay nada definitivo, todo está en desarrollo, en movimiento. Lo que sí está claro es que es necesaria una crítica a la colonialidad del poder en todas sus dimensiones. Esto de crítica tiene que entenderse bien; hay al menos dos problemas que la rodean. Primero, la particularidad de ir a contrapelo de lo convencional, lo que la hace doblemente difícil, para criticar algo es necesario conocerlo a profundidad, con esto quiero decir que el objeto de crítica es lo que mejor debemos conocer; no es rechazarlo, es comprenderlo para criticarlo, esto cuesta, porque parece que se hace lo contrario, se critica rechazándolo. El otro problema es que el giro decolonial es atacado por los sectores más conservadores de la academia y del establishment que se amparan en el positivismo; lo interesante sería un debate confrontando perspectivas, pero estos más bien hacen 
mofas de los enunciados y tesis del giro decolonial, a pesar de que los problemas que se tratan desde esta perspectiva son los referentes a las diversas afecciones que sufren los seres humanos por una determinada forma de organizar la vida en el planeta.

Ya hemos pasado lista brevemente a algunos de los aspectos relevantes, no queriendo decir que los hemos agotado todos, pero si para dejar una idea de lo que es o trata el giro decolonial, un concepto aún en construcción. Hay que hurgar hasta el fondo como la labor de arqueología para encontrar nuevas formas de cuestionar sobre los viejos problemas que persisten.

No se pueden negar los aportes del pensamiento europeo y norteamericano en los estudios de esta naturaleza, el giro decolonial no tiene que ser una negación total de todo lo moderno - occidental, sino una invitación a leerlo con mayor atención. Hay que leer en clave decolonial los textos canónicos, así es más enriquecedor que el simple hecho de condenarlos por ser eurocéntricos y cosas como esa que no aportan mucho. En tiempos difíciles, de convulsiones globales lo que nos queda es:

Saldar cuentas con nuestro pasado es como abrir las puertas hacia nuestro propio futuro hasta ahora inconcluso, es como liberar las fuerzas del pasado en el que el presente, para que nos abra hacia otros futuros radicalmente distintos y auténticamente nuevos. Es como inaugurar otro tiempo, otro futuro, es decir, otro mundo en que todos puedan vivir dignamente. (Bautista, 2014, pp. 252-253)

Estas serían, entre otras cuestiones, las tareas del giro decolonial en el siglo XXI.

\section{Referencias}

Bautista, J. J. (2012). Hacia la descolonización de la ciencia social latinoamericana. Cuatro ensayos metodólogicos y epistemológicos. La Paz: Rincón Ediciones.

Bautista, J. J. (2014). ¿Qué significa pensar desde América Latina? Madrid: Akal.

Bhabha, H. (2002). El lugar de la cultura. Buenos Aires: Manantial. 
Bidaseca, K. (2016). Genealogías críticas de la colonialidad en América Latina, África, Oriente. Buenos Aires: UNSAM / IDAES / CLACSO.

Boidin, C. y Hurtado López, F. (2010). La philosophie de la libération et le courant décolonial. Cahiers des Amériques latines, 62, 17-22.

Brah, A. (2011). Cartografias de la diáspora. Identidades en cuestión. Madrid: Traficantes de Sueño.

Castro-Gómez, S. y Grosfoguel, R. (2007). El giro decolonial. Reflexiones para una diversidad epistémica más allá del capitalismo global. Bogotá: Siglo del Hombre Editores / IESCO-UC / Pontificia Universidad Javeriana.

Chakrabarty, D. (2008). Al margen de Europa. El pensamiento poscolonial y diferencia histórica. Barcelona: Ensayo Tusquets.

Chakrabarty, D. (2008). La historia subalterna como pensamiento político. En S. Mezzadra (Ed.), Estudios poscoloniales. Ensayos fundamentales, (pp. 145-166). Madrid: Traficantes de sueños.

Colón, C. (1997). Textos y documentos completos. Nuevas cartas. (C. Varela, y J. Gil, Edits.) Madrid: Alianza.

Curiel, O. (2015). Construyendo metodologías feministas desde el feminismo decolonial. En Otras formas de (re) conocer (pp. 4560). Gipuzkoa: UPV / HEGOA / SIMRF.

Davis, A. (2005). Mujer, raza y clase. Madrid: Akal.

Davis, A. (2016). Democracia de la abolición. Prisiones, racismo y violencia ( $1^{\mathrm{a}}$ ed.). Madrid: Trotta.

De Sousa Santos, B. y Meneses, M. P. (2014). Espistemologías del sur (Perspectivas). Madrid: Akal.

De Sousa Santos, B. (2009). Una epistemología del sur. La reinvención del conocimiento y la emancipación social. México: CLACSO / Siglo XXI.

Dos Santos, T. (2002). La teoria de la dependencia: Balance y perspectivas. México: Plaza \& Janés.

Dussel, E. (1974). Historia de la iglesia en América Latina. Coloniaje y liberación 1492 / 1973 ( ${ }^{\mathrm{a}}$ ed.). Barcelona: Nova Terra.

Dussel, E. (1980). La pedagógica latinoamericana. Bogota: Nueva América.

Dussel, E. (2007). El primer anti-discurso filosófico de la modernidad: La crítica de la expansión colonial europea por Bartolomé de las 
Casas. En Política de la liberación. Historia mundial y crítica (199-205). Madrid: Trotta.

Dussel, E. (2011). Filosofia de la liberación. México: Fondo de Cultura Económica.

Dussel, E. (2015). Filosofias del sur. Descolonización y transmodernidad. México: Akal.

Elliot, J. (2015). El viejo y el nuevo mundo (1492-1650) ( ${ }^{\text {a }}$ ed.). Madrid: Alianza.

Freire, P. (1992). Pedagogía del oprimido. Madrid: Siglo XXI.

Grosfoguel, R. (2007). Los dilemas de los estudios étnicos estadounidenses: Multiculturalismo identitario, colonización disciplinaria y epistemologías decoloniales. Universitas Humanística, pp. $35-47$.

Grosfoguel, R. (2016). What is Racism?. Journal of World-Systems Research, 22, 9-15.

Guha, R. y Spivak, G. (1988). Selected Subaltern Studies. New York: Oxford University Press.

Hall, S. (2008). ¿Cuándo fue lo postcolonial? Pensar al límite. En S. Mezzadra (Ed.), Estudios postcoloniales Ensayos fundamentales (pp. 121-144). Madrid: Traficantes de Sueño.

Han, B. C. (2014). Psicopolitica. ( ${ }^{a}$ ed.). Barcelona: Herder.

Hanna, M., Harford, J. y Saldívar, J. (2016). Junot Díaz and the Decolonial Imagination. Durham: Duke University Press .

Heidegger, M. (1989). La autoafirmación de la universidad alemana. El rectorado, 1933-1934. Entrevista del Spiegel. Madrid: Tecnos.

Hill, P. (2000). Black Feminist Thought ( ${ }^{\text {a }}$ ed.). New York and London: Routledge.

Hurtado López, F. (2010). Pensée critique latino-américaine:de la philosophie de la libération au tournant décolonial. Cahiers des Amériques latines, 62, 23-34.

Insausti, X. (2015). Ideas para una filosofía crítica en el siglo XXI. En X. Insausti, M. Nogueroles y J. Vergara (Edits.), Nuevos diálogos de pensamiento crítico (pp. 119-131). Madrid: UAM / UPV / RIPC.

Jabardo, M. (2012). Feminismos negros. Una antología. Madrid: Traficantes de Sueño. 
Lander, E. (2000). La colonialidad del saber: Eurocentrismo y ciencias sociales. Perspectivas latinoamericanas. (1era ed.). Buenos Aires: CLACSO / UNESCO.

León Portilla, M. (2005). Visión de los vencidos: Relaciones indígenas de la conquista. México: Universidad Nacional Autónoma de México.

Levinas, E. (2005). Humanismo del otro hombre. México: Siglo XXI. Lugones, M. (2008). Colonialidad y género. Tabula Raza, 9, 73-101.

Maldonado-Torres, N. (2006). Césaire's Gift and the Decolonial Turn. Radical Philosophy Review, 9(2), 111-138.

Maldonado-Torres, N. (2007). Sobre la colonialidad del ser: Contribuciones al desarrollo de un concepto. En S. Castro-Gómez \& R. Grosfoguel (Edits.), El giro decolonial. Reflexiones para una diversidad epistémica más allá del capitalismo global (pp. 127167). Bogotá: Siglo del Hombre Editores / IESCO-UC / Pontificia Universidad Javeriana.

Maldonado-Torres, N. (2008). Against War: Views from the Underside of Modernity. Durham: Duke University Press.

Maldonado-Torres, N. (2011). El pensamiento filosófico del "giro descolonizador". En E. Dussel, E. Mendieta y C. Bohórquez (Edits.), El pensamiento filósofico latinoamericano, del Caribe y "latino" 1300-2000 (pp. 683-697). México: Siglo XXI.

Mignolo, W. (2007). El pensamiento decolonial: Desprendimiento y apertura. Un manifiesto. En El giro decolonial. Reflexiones para una diversidad epistémica más allá del capitalismo global (pp. 25-46). Bogotá: Siglo del Hombre Editores / IESCO-UC / Pontificia Universidad Javeriana.

Mignolo, W. (2014). Género y descolonialidad. Argentina. ( $2^{\mathrm{a}}$ ed.). Buenos Aires: Ediciones del Signo / Duke University.

Moraña, M., Dussel, E. y Jáuregui, C. (2008). Coloniality at Large: Latin America and the Postcolonial Debate. Durham: Duke University Press.

Pulido, L. (2016). Problematizando al eurocentrismo en la época transnacional: El tutelaje y la teoría de la decolonialidad. Cuadernos Inter.c.a.mbio sobre Centroamérica y el Caribe, 13, 111-134.

Quijano, A. (2000). Colonialidad del poder y clasificación social. Journal of World-Systems Research, I(2), 342-386. 
Robindson, C. (2000). Black Marxism. Chape Hill \& London: The University of North Carolina Press.

Salas, M. y Valle, E. (2002). Cultura, poder e identidad; la dinámica y trayectoria de los intelectuales chicanos en los Estados Unidos. En D. Mato (Ed.), Estudios y otras prácticas intelectuales latinoamericanas en cultura y poder. Buenos Aires: CLACSO.

Sandoval, C. (2000). Methodology of the Oppressed. Minneapolis \& London: University of Minnesota Press.

Spivak, G. (1998). ¿Puede hablar el sujeto subalterno?. Orbis Tertius, $6,175-235$.

Tuhiwai, L. (1999). Decolonizing Methodologies. Research and Indigenous Peoples. London \& New York: Zed Books Ltd / University of Otago Press.

Wallerstein, I. (1974). The Modern World System I: Capitalist Agriculture and the Origins of the European World-Economy in the Sixteenth Century. New York: Academic Press. 